\title{
Incidentally discovered pelvic paraganglioma: A case report
}

\author{
Vittorio Imperatore ${ }^{1}$, Massimiliano Creta ${ }^{1}$, Sergio Di Meo ${ }^{1}$, Roberto Buonopane ${ }^{1}$, Lorenzo Spirito ${ }^{2}$, \\ Ferdinando Fusco $^{2}$, Nicola Longo ${ }^{2}$, Nicola Rosario Forte ${ }^{3}$, Vincenzo Mirone ${ }^{2}$ \\ ${ }^{1}$ Operative Unit of Urology, Buon Consiglio Hospital - Fatebenefratelli, Naples, Italy; \\ ${ }^{2}$ Urological Clinic, Federico II University of Naples, Naples, Italy; \\ ${ }^{3}$ Operative Unit of Pathological Anatomy, Fatebenefratelli Hospital, Benevento, Italy.
}

\begin{abstract}
Summary Paragangliomas (PGL) are rare neuroendocrine tumors of the autonomic nervous system originating from paraganglia. Although PGL may arise at any site where physiologic paraganglionic tissue exists, the localization in the small pelvis is extremely rare. PGL may be hormonally active and release surplus catecholamines into the blood or inactive. The asymptomatic cases pose a diagnostic dilemma. We describe the case of an asymptomatic PGL arising in the small pelvis sidewall presenting as an incidentally discovered asymptomatic mass in a male subject.
\end{abstract}

KEY WORDS: Magnetic Resonance Imaging; Paraganglioma.

Submitted 1 August 2017; Accepted 16 September 2017

\section{INTRODUCTION}

Paragangliomas (PGL) are rare tumors of the autonomic nervous system that originate from paraganglia which are neuroendocrine organs characterized by catecholamine and peptide-producing cells deriving from the neural crest (1). The role of normal paraganglia is homeostasis, either by acting directly as chemical sensors or by secreting catecholamines in response to stress (1). The paraganglionic system comprises the adrenal medulla and the extra-adrenal paraganglia.

In 2004 the World Health Organization defined a pheochromocytoma as an intraadrenal PGL, whereas related tumors of extraadrenal sympathetic or parasympathetic paraganglia are defined as extraadrenal PGL (1).

Although extra-adrenal PGL may arise at any site where physiologic paraganglionic tissue exists, the localization in the small pelvis is extremely rare. From a functional point of view PGL may be hormonally active or inactive. Functional PGL result in symptoms of excess catecholamine production. Non-functional PGL pose a significant diagnostic challenge. We describe the case of an asymptomatic PGL of the small pelvis sidewall incidentally discovered in a male patient.

\section{Case report}

A 69-year-old man came to our attention for an incidentally discovered $32 \mathrm{~mm}$ hypoechoic lesion at the level of the left paravesical space on abdominal ultrasound imaging. His past medical history was relevant for simple prostatectomy performed 1 year before. His Prostate Specific Antigen value was 2.9 ng/dL. Physical examination including digital rectal examination was unremarkable. His arterial blood pressure and heart rate were within the normal ranges. He denied systemic symptoms, lower urinary tract symptoms, pain, and hematuria. A computed tomography of the abdomen revealed the presence of a $38 \mathrm{~mm}$ nodular mass located at the level of the left obturator space characterized by internal inhomogeneity and an intense peripheral enhancement in the portal phase with rapid washout. No significant abnormalities of other abdominal organs were detected. A Magnetic Resonance Imaging (MRI) with contrast medium of the pelvis confirmed the presence of an inhomogeneous $38 \times 35 \mathrm{~mm}$ extraperitoneal mass located into the left obturator space in the proximity of the vesicoprostatic junction characterized by an intense contrast enhancement in the arterial phase and expansive growth pattern (Figure 1).

Although suggestive of probable benign disease, radiological findings were inconclusive, and an exploratory laparotomy with excision of mass was planned.

The procedure was technically challenging due to the adherence of the mass to the vascular structures of the

\section{Figure 1.}

Transverse (A) and coronal (B) MRI section of the pelvis showing a well-defined, rounded, heterogeneous enhancing mass measuring 3.8 $x 3.5 \mathrm{~cm}$ at the level of the left obturator space (arrow). No regional lymphadenopathy or invasion of the adjacent structures were evident.
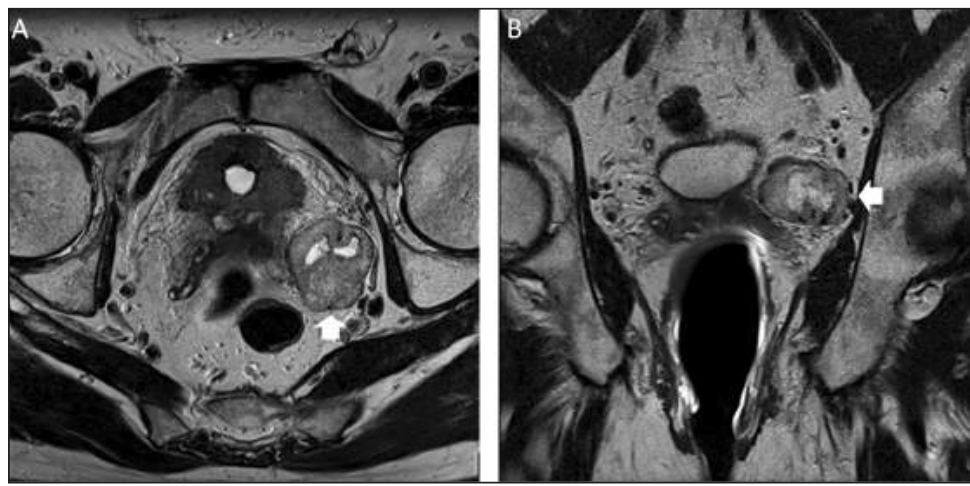
small pelvis and to the ureter. The post-operative course was uneventful and the patient was discharged home on post-operative day 5. Histological examination revealed a proliferation of neoplastic cells arranged in solid nests. Immunohistochemistry revealed positivity for synaptophysin and S-100 which was located at the periphery of the clusters of cells.

Immunostaining for CK 7, CK 20 and prostate specific antigen was negative. The Ki 67 proliferative index was $7 \%$. The histological and immunohistochemical findings were compatible with the diagnosis of PGL. Post-operative staging with whole body contrast enhanced computed tomography showed no residual tumor or distant metastases. 24-h urinary excretion of catecholamines, were within normal limits.

\section{Discussion}

Extra-adrenal PGL are rare tumors that can occur in isolation or as a part of multisystem disorders such as multiple endocrine neoplasia, neurofibromatosis or von Hippel-Lindau disease (1). Clinical presentation of extraadrenal PGL is highly variable and depends on the location, the size, and the functional status of the tumor. PGL may present at all ages but the peak incidence is around 30-50 years. Although most PGL are benign, the probability of malignant PGL reaches up to $25 \%$ depending on the presence of genetic mutations. Commonly, extraadrenal PGL arise from the para-aortic region at the level of the renal hila, the organ of Zukerkandl, the thoracic paraspinal region, head, and neck (2). Rarely, PGL occur in the small pelvis and may arise from the bladder, the seminal vesicles, or the pelvis sidewall (2). If the tumor is hormonally active, the patient may experience symptoms and signs of catecholamine overproduction, such as hypertension, headaches, palpitations, sweating, tachycardia, and anxiety (2). Symptoms of excess catecholamine production, as well as elevated urine vanillylmandelic acid levels and serum and urine norepinephrine levels, are highly diagnostic for PGL (3).

Unfortunately, most cases of PGL arising in the small pelvis are nonfunctional and the diagnosis is made in cases of advanced stage, nonspecific symptoms or incidentally (2). To our knowledge, we described the second case of pelvic PGL arising from the pelvis sidewall. MRI represents an optimal diagnostic modality in cases of uncommon pelvic retroperitoneal masses in adults thanks to the multiplanar capability and the high-resolution imaging thus permitting better tumor localization and internal characterization (3-5). Moreover, MRI provides useful data to plan surgical resection (3). At MRI, PGL appear as areas of low T1 and high T2 signal intensity and show avid enhancement following the administration of gadoliniumbased contrast material (3). The differential diagnosis for PGL in the pelvic retroperitoneum includes nerve sheath tumors, hypervascular lymphadenopathy (metastases or Castleman disease), and hypervascular soft-tissue sarcomas (3). Percutaneous biopsy of a hypervascular pelvic retroperitoneal mass may be harmful since it can result in hypertensive crisis or even death (3). In the present case, the pre-operative suspicious of PGL was very low due to the lack of specific symptoms. The definitive diagnosis is made by histopathological examination of the removed tumor and is confirmed by immunohistochemistry. Treatment options for PGL include, surgery, radiotherapy, chemotherapy, radiofrequency ablation and cryoablation. The standard treatment for localized or locally advanced PGL is surgical resection of the tumor (2). Surgical tumor excision is in most cases curative. However, PGL can represent a surgical challenge due to their tight relation to vessels. The prognosis of extra-adrenal PGL is a matter of debate as the definitive diagnosis of malignancy is difficult. Tumor size $>5 \mathrm{~cm}$, occurrence of invasion or metastatic disease and hormonal activity are considered predictors of malignancy. Although uncommon, late tumor recurrences in the surgical bed or systemic may occur and close follow-up is required.

\section{Conclusions}

PGL are rare tumors that should be included in the differential diagnosis of incidentally found pelvic hypervascular masses. Hormonally inactive PGL pose diagnostic challenges and surgical excision requires extensive surgical skills.

\section{REFERENCES}

1. Lefebvre M, Foulkes WD. Pheochromocytoma and paraganglioma syndromes: genetics and management update. Curr Oncol. 2014; 21:e8-e17.

2. Sahdev A, Sohaib A, Monson JP, et al. CT and MR imaging of unusual locations of extra adrenal paragangliomas (pheochromocytomas) Eur Radiol. 2005; 15:85-92.

3. Shanbhogue AK, Fasih N, Macdonald DB, et al. Uncommon primary pelvic retroperitoneal masses in adults: a pattern-based imaging approach. Radiographics. 2012; 32:795-817.

4. Creta M, Mirone V, Di Meo S, et al. A rare case of male pelvic squamous cell carcinoma of unknown primary origin presenting as perineal abscess and urethral stenosis. Arch Ital Urol Androl. 2017;89:154-155

5. Cennamo P, Montuori N, Trojsi G, et al. Biofilms in churches built in grottoes. Sci Total Environ. 2016; 543:727-38.

\author{
Correspondence \\ Vittorio Imperatore, MD (Corresponding Author) \\ v.imperatore@alice.it \\ Massimiliano Creta, MD \\ max.creta@gmail.com \\ Sergio Di Meo, MD \\ s.dimeo72@gmail.com \\ Roberto Buonopane, MD \\ robertobuonopane@libero.it \\ Operative Unit of Urology, Buon Consiglio Hospital - Fatebenefratelli, \\ Naples, Italy \\ Via A. Manzoni, 220, 80123 Napoli \\ Lorenzo Spirito, MD - Ferdinando Fusco, MD - Nicola Longo, MD \\ Vincenzo Mirone, MD \\ Urological Clinic, Federico II University of Naples, Naples, Italy \\ Via S.Pansini, 5, 80131 Napoli \\ Nicola Rosario Forte, MD \\ Operative Unit of Pathological Anatomy, Fatebenefratelli Hospital, \\ Benevento, Italy \\ Viale Principe di Napoli, 14, 82100 Benevento
}

